\title{
Magnetic resonance imaging of the subthalamic nucleus for deep brain stimulation
}

\author{
Arjun S. Chandran, MBBS, ${ }^{1}$ Michael Bynevelt, BHB, MBChB, FRANZCR, ${ }^{2,3}$ and \\ Christopher R. P. Lind, FRACS, 1,3
}

\begin{abstract}
Departments of ${ }^{1}$ Neurosurgery and ${ }^{2}$ Radiology, Sir Charles Gairdner Hospital, and ${ }^{3}$ School of Surgery, University of Western Australia, Perth, Western Australia, Australia
\end{abstract}

\begin{abstract}
The subthalamic nucleus (STN) is one of the most important stereotactic targets in neurosurgery, and its accurate imaging is crucial. With improving MRI sequences there is impetus for direct targeting of the STN. High-quality, distortion-free images are paramount. Image reconstruction techniques appear to show the greatest promise in balancing the issue of geometrical distortion and STN edge detection. Existing spin echo- and susceptibility-based MRI sequences are compared with new image reconstruction methods. Quantitative susceptibility mapping is the most promising technique for stereotactic imaging of the STN.
\end{abstract}

http://thejns.org/doi/abs/10.3171/2015.1.JNS142066

KEY WORDS subthalamic nucleus; magnetic resonance imaging; deep brain stimulation; functional neurosurgery

$\mathrm{F}$ UTURE progress to refine targets for deep brain stimulation (DBS) is critically dependent on precise placement and verification of electrodes in the brain. No stereotactic target is of more clinical importance or more nuanced regarding its imaging than the subthalamic nucleus (STN). The STN is difficult to image because of its biconvex shape, small size, oblique spatial orientation in 3 planes, and its close proximity to the substantia nigra $(\mathrm{SN}) .^{3}$ A high level of accuracy is required as the motor region of the STN is posterolateral, and unwanted stimulation side effects may occur as a result of direct stimulation or spread to the anteromedial nonmotor area and the adjacent corticospinal tract. ${ }^{9,18,31,56}$ Current imaging of STN borders is difficult.

Targeting of the STN has historically been performed indirectly; the neurosurgeon predicts the location of the STN based on coordinates derived from atlases. These coordinates have often been related to the midcommissural point, which was derived from x-ray ventriculography but also from CT and MRI. Retaining this indirect, coordinate-based approach has been attractive to neurosurgeons. Experience in using this approach has been handed down from the past and can be used even with modern noninvasive imaging, and targeting preferences can be easily com- municated between surgeons. The problem with indirect targeting is that different patients have different STN sizes, shapes, and positions..$^{38}$ Direct targeting is an attempt to locate the STN in each individual patient.

Poor image quality on traditional 1.5 tesla $(\mathrm{T})$ scanners and primitive pulse sequences that do not provide adequate contrast at the level of the STN have limited the progression of direct targeting in some centers. Also hindering the development of stereotactic MRI are concerns about image distortion with higher field strength; traditional stereotactic head frames and localizers are too bulky to permit the use of high-quality, multichannel MRI head coils.

Despite these challenges, higher field strength scanners and new pulse sequences have improved image quality to a point that some groups have adopted direct targeting of the STN, based on MRI. ${ }^{3,9,37}$ Difficulty delineating the STN and spatial inaccuracy has resulted in reluctance from others to abandon adjuncts such as CT/MRI merge and intraoperative electrophysiology.

Traditionally, T2-weighted imaging (T2WI) has been used to plan for direct targeting of the STN using a variety of imaging parameters (Fig. 1). ${ }^{9}$ Although T2WI is useful, it is difficult to clearly delineate the STN from the SN and, to a lesser extent, the zona incerta (ZI). ${ }^{22,32,37}$

\footnotetext{
ABBREVIATIONS CNR = contrast-to-noise ratio; DBS = deep brain stimulation; $D T I=$ diffusion tensor imaging; FA = fractional anisotropy; FGATIR = fast gray matter acquisition T1 IR; FLAIR = fluid attenuated IR; FLASH = fast low-angle shot; FSE = fast spin echo; GRE = gradient echo; IR = inversion recovery; NSA = number of signal averages; PSIR = phase-sensitive IR; QSM = quantitative susceptibility mapping; SE = spin echo; SN = substantia nigra; SNR = signal-to-noise ratio; STIR = short tau IR; STN = subthalamic nucleus; SWI = susceptibility-weighted imaging; SWPI = susceptibility-weighted phase imaging; $\mathrm{T}=$ tesla; $\mathrm{T} 2 \mathrm{WI}=\mathrm{T} 2$-weighted imaging; $\mathrm{T} 2{ }^{*} \mathrm{WI}=$ T2-weighted magnitude imaging; $\mathrm{TI}=$ inversion time; TR = relaxation time; $\mathrm{ZI}$ = zona incerta.
}

SUBMITTED September 5, 2014. ACCEPTED January 22, 2015.

INCLUDE WHEN CITING Published online August 21, 2015; DOI: 10.3171/2015.1.JNS142066. 


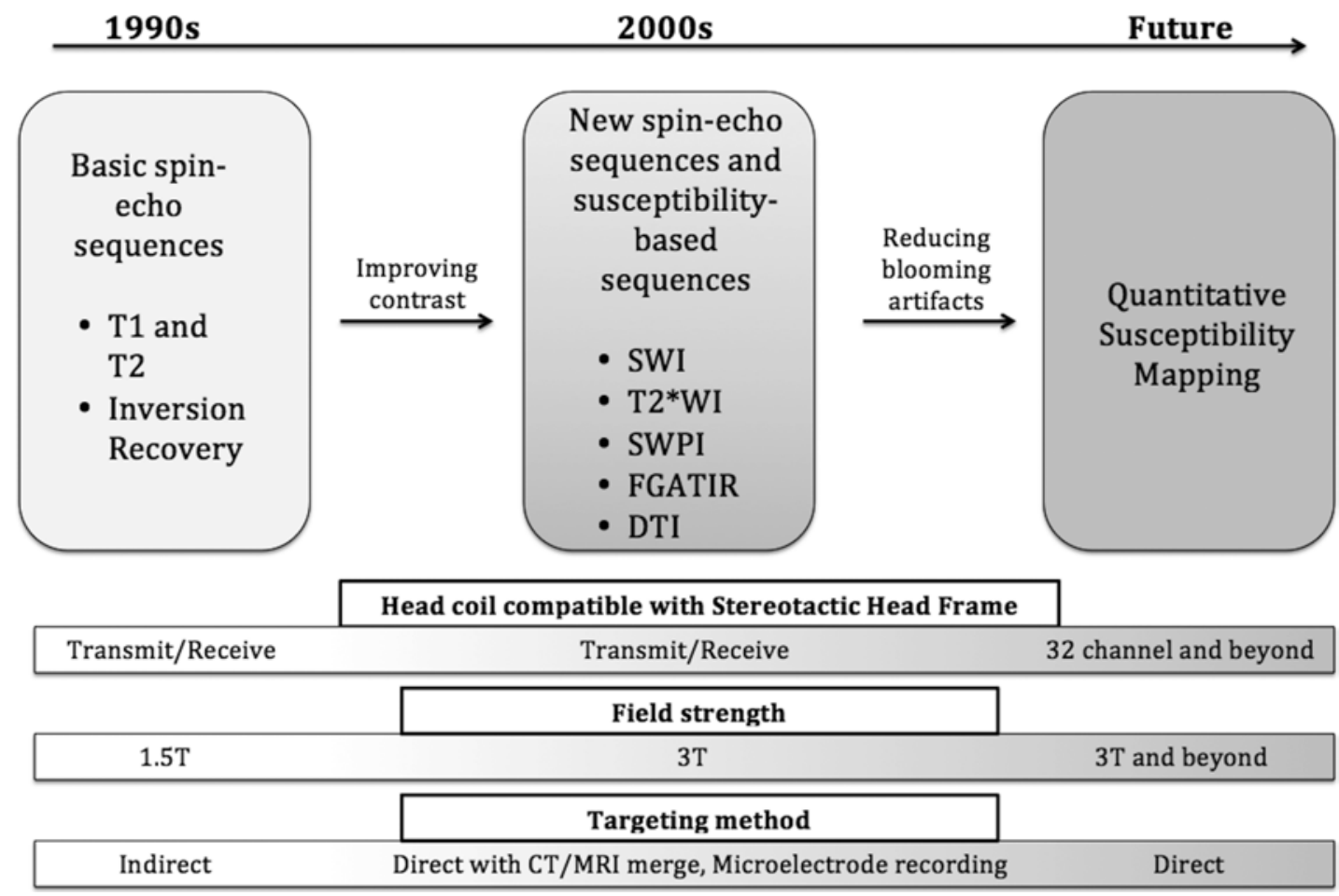

FIG. 1. Schematic overview showing the evolution of STN imaging and targeting methods since the 1990s. Pulse sequences, head coil availability, field strength, and targeting methods are placed in a historical context.

Native and reconstructed MRI pulse sequences are being investigated to more clearly define the STN. Broadly speaking, these sequences can be separated into spin echo (SE) methods, dependent on the precession of water molecules, and susceptibility-based methods that take into account the underlying composition of the brain tissue.

The native SE methods substantiated by the most research evidence are T2WI, inversion recovery (IR), and diffusion tensor imaging (DTI) utilizing the fractional anisotropy (FA) parameter. The native susceptibility-based techniques with the most evidence include susceptibilityweighted imaging (SWI) and T2-weighted magnitude imaging (T2*WI). Image reconstruction methods such as susceptibility-weighted phase imaging (SWPI) and quantitative susceptibility mapping (QSM) have also shown promise at reducing image distortion.

Neurosurgeons need to know which sequences will provide the best visualization of the STN with the least amount of geometrical distortion to optimize stereotactic surgery.

\section{Methods}

A systematic search of PubMed and Google Scholar databases for full-length articles published up until March 1, 2014, was performed. Search expressions were built using combinations of predefined medical subject heading terms and comprised "Subthalamic Nucleus AND Magnetic Resonance Imaging," "Magnetic Resonance Imaging AND Deep Brain Stimulation," "Subthalamic Nucleus AND Magnetic Resonance Imaging AND Deep
Brain Stimulation.” Abstracts of resulting searches were screened, and those papers pertaining directly to STN visualization using MRI were read in full. Reference lists in those papers were screened and further papers identified. Based on this literature review, T2WI, SWI, SWPI, T2*WI, IR imaging, DTI, and QSM sequences were identified as the most thoroughly investigated sequences and those with the most data for accurate visualization of the STN.

Study inclusion criteria were 1) publications in the English language in a peer-reviewed journal or book, 2) articles pertaining directly to visualization of the STN using MRI, 3) pulse sequence parameters were well defined and reproducible, and 4) articles pertaining to T2WI, SWI, SWPI, T2*WI, IR imaging, DTI, and QSM sequences.

\section{MRI Sequences}

\section{Spin Echo Techniques}

T2-Weighted Imaging. The STN appears hypointense on T2WI of the brain (Fig. 2), and anatomical studies have suggested this is secondary to relative high intrinsic iron content of the tissue. T2WI is dependent on the T2 relaxation of brain tissue, i.e., the decay of the transverse magnetization measured in SE sequences. The effect of the higher iron content causes hastening of the transverse magnetization decay, with shortening of the tissue T2 relaxation time (TR). Consequently there is less signal in the nuclei than the surrounding tissue. ${ }^{14}$

T2WI is the most studied imaging sequence and remains one of the most commonly used for STN targeting. 

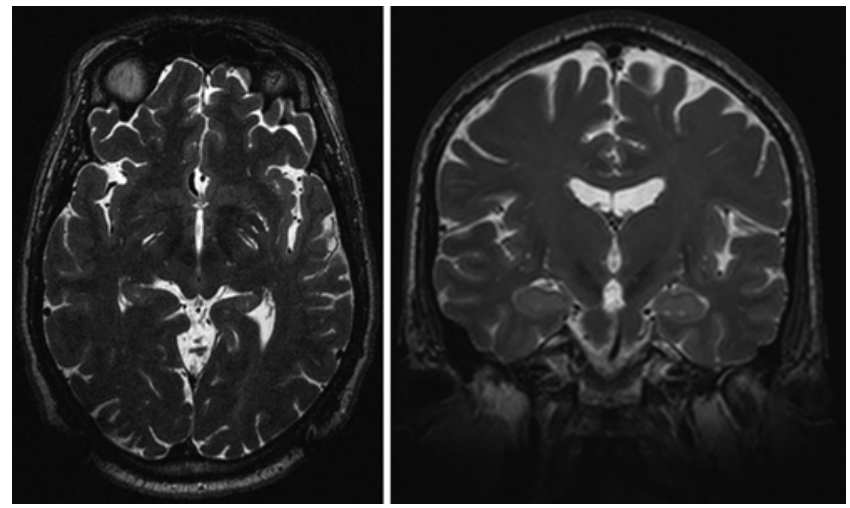

FIG. 2. Preoperative axial (left) and coronal (right) 3-T T2-weighted FSE planning MRI through the center of the STN obtained in a patient wearing the Leksell stereotactic frame G. Image parameters as described in Thani et al. ${ }^{55}$

T2WI has yielded mixed results in portraying the STN, particularly at low field strength $(1.5 \mathrm{~T})$, with image distortion and poor delineation from its surrounding gray matter structures. Ashkan et al. ${ }^{3}$ demonstrated by imaging 29 consecutive patients that T2WI provided a more accurate preoperative method of localization of the STN than atlas-based targeting methods. Other groups provided data showing image distortion and low resolution may make direct targeting using T2WI alone problematic.,59 Others have shown good clinical results using this sequence while scanning a patient who is anesthetized and undergoing direct anatomical surgical techniques. ${ }^{38}$

Distortion remains one of the most significant challenges in MRI imaging of the STN. MRI distortion alone in T2 fast SE (FSE) sequences has been shown to cause a total error of up to $2.4 \mathrm{~mm}$, limiting the accuracy of MRI in targeting very small structures..$^{59}$ These distortions have been noticed particularly at the peripheries of the target structures, obscuring tissue borders. ${ }^{34}$ This distortion appears to be amplified when using T2 FSE sequences in combination with a stereotactic head frame; however, more recent studies have suggested that this distortion may not be as significant as hypothesized. ${ }^{50}$

Further, given that contrast resolution of the STN on $\mathrm{T} 2$ sequences is dependent on tissue iron content, the clear demarcation of STN borders from surrounding iron-laden tissues, including the SN and the red nucleus, is difficult. The most difficult border to visualize remains the anteromedial and ventral aspects abutting the SN.6,12 Starr et al. reported after looking at 76 implanted DBS leads using 1.5-T T2 FSE sequences that the STN was visualized directly in $92 \%$ of cases; however, it was rare to visualize all borders of the STN with perfect precision. ${ }^{51}$ This result is difficult to interpret given distortion from the in situ DBS lead, which itself may have obscured the STN border.

Poor image quality, limited contrast resolution, and distortion are the reasons a number of neurosurgeons choose to use microelectrode recording intraoperatively. Unfortunately, microelectrode recording has some inherent disadvantages, including increased operative time and the possible need for multiple passes, which may increase the risk of bleeding and infection. ${ }^{28}$
The utilization of high-field (3-T) MRI facilities, which have become widely available, is thought to be one reason this method is improving STN resolution. Higher field strength theoretically improves the signal-to-noise ratio (SNR) proportional to the higher field strength. ${ }^{11}$ It also allows for increased T1 TR and decreased T2 TR, which leads to improved image resolution and/or shortened imaging time. ${ }^{30}$ Direct comparison of 1.5-T and 3-T T2 sequences demonstrated better demarcation of the STN using 3-T MRI. ${ }^{11}$ Analysis of 3-T T2WI in 20 patients demonstrated sufficient resolution to accurately visualize the STN in 3 dimensions and correlated well with STN measurements in cadaveric specimens. ${ }^{37}$ Further studies have suggested that even higher field strength $(7 \mathrm{~T}$ and $9.4 \mathrm{~T}$ ) may further improve delineation of the $\mathrm{SN}$ from the STN. ${ }^{1,33}$ Despite these promising results, geometrical distortion at these higher field strengths is expected to increase, which may compromise the potential gain in contrast resolution.

Direct comparison of T2-weighted FSE sequences with SWI, T2*WI, phase-sensitive IR (PSIR), IR-FSE, and T1 at $1.5 \mathrm{~T}$ showed that $\mathrm{T} 2 \mathrm{FSE}$ performed considerably better than other SE sequences including PSIR, IR-FSE, and T1 for imaging the STN. ${ }^{36}$

In comparison with susceptibility-based techniques, however, SWI and T2*WI performed better than T2WI, with a statistically significant increase in contrast-tonoise ratio (CNR) using the newer SWI technique. While T2*WI CNR was also higher, this did not attain significance. These improvements in image quality with SWI and T2*WI are amplified at $3 \mathrm{~T}$. Direct comparison of T2 FSE with QSM at $3 \mathrm{~T}$ showed that the CNR of QSM was a median of 6.4 and 10.7 times higher for differentiation of the STN from the ZI and SN, respectively. ${ }^{29}$ These results require verification in patients undergoing DBS with a head frame in situ; however, early indications suggest newer pulse sequences in which contrast is more directly dependent on intrinsic tissue susceptibility and with lower geometrical distortion may soon supersede T2WI for direct targeting of the STN.

Diffusion Tensor Imaging. DTI techniques allow in vivo examination of white matter fiber tracts, providing both the location and relationship information as well as predicting abnormal anatomy. ${ }^{5,35,39,47}$ This is of use in DBS to minimize side effects caused by collateral activation of adjacent white matter tracts. Shields et al. demonstrated, for example, that eye deviation during STN stimulation is likely due to activation of the anterior limb of the internal capsule leading to the frontal eye fields. ${ }^{48}$ Traditionally, avoidance of these pathways has been achieved either by waking the patient to assess for activation of these surrounding pathways or using microelectrode recording. The importance of DTI in DBS surgery is debatable given it has traditionally been used in cases of altered anatomy, e.g., tumors which shift or involve white matter pathways. The benefit of using this technique in cases with normal anatomy is unknown.

DTI has previously been used in direct targeting of white matter tracts, ${ }^{19}$ and some studies have suggested that the use of the FA parameter may help DBS surgeons identify tracts surrounding the STN. Using DTI with FA, 
white matter tracts related to the STN are mapped, showing relationships with the primary motor cortex, premotor cortex, and frontal eye fields. ${ }^{4}$ These tracts in the subthalamic region have been shown to be more readily identifiable using DTI and FA than T1-weighted imaging and T2WI ${ }^{46}$ It is likely that DTI with FA would need to be used as an adjunct to other MRI sequences rather than as a primary targeting tool to gain the gray matter anatomical detail necessary for direct targeting of the STN during DBS.

Inversion Recovery. IR sequences are a broad range of sequences used to enhance contrast by selectively suppressing certain tissues with a specific composition and are commonly used by neurosurgeons worldwide for STN targeting. ${ }^{15}$ The application of multiple IR sequences have been investigated for imaging the STN, including fluid attenuated IR (FLAIR), short tau IR (STIR), PSIR, and newer experimental techniques including fast gray matter acquisition T1 IR (FGATIR).

FLAIR sequences (Fig. 3) have been commonly used by neurosurgeons for direct targeting. The STN appears hypointense, and gray matter contrast is appreciable; however, delineation from the SN is difficult. Recent comparison of FLAIR with newer susceptibility sequences showed limited delineation of the STN on axial sequences at $3 \mathrm{~T}$. SWI had a statistically significant higher CNR than FLAIR. ${ }^{22}$ The prevalence of use of FLAIR among neurosurgeons may be due to the ready availability and rapid acquisition time, but, perhaps more importantly, it gives the STN an appearance of large mass. There are difficulties, however, with border delineation, especially adjacent to the SN. Interestingly, there is limited evidence comparing FLAIR sequences with other SE or newer susceptibility sequences despite its anecdotal prevalence in use.

The PSIR method has been shown to provide good SNR and CNR to the area of the STN, even with thin slices. ${ }^{21,52}$ This technique remains one of the few that has been verified with a head frame (Leksell stereotactic frame G [Elekta]) and has an estimated geometrical distortion of less than $1 \% .^{21}$ This is particularly advantageous to neurosurgeons using stereotactic MRI rather than stereotactic CT/MRI merge techniques.$^{54}$ It also represents one of the few imaging sequences that have had sequence parameters compared scientifically to find optimal parameters for visualization of the STN. It was determined that TR and inversion time (TI) of $4000 \mathrm{msec}$ and $200 \mathrm{msec}$, respectively, provided the best visualization scores for the STN (compared with TRs of 2000, 3000, and $5000 \mathrm{msec}$ and TIs of 100-900 msec). ${ }^{21}$

Direct comparison of PSIR with T2WI, T2*WI, and SWI has shown PSIR has poorer CNR. ${ }^{36}$ Despite this, because of the scarcity of data regarding geometrical accuracy of SWI and T2*WI, PSIR remains a viable and desirable option for accurate direct targeting of the STN.

T1 STIR sequences have also been investigated, with a suggestion that they could be used in conjunction with $\mathrm{T} 2$ FSE sequences to view all the borders of the STN. Direct comparison between T2 FSE and STIR in healthy subject populations demonstrated higher CNR between the STN and SN using STIR sequences. The lateral and superior borders with the internal capsule and ZI, respectively,
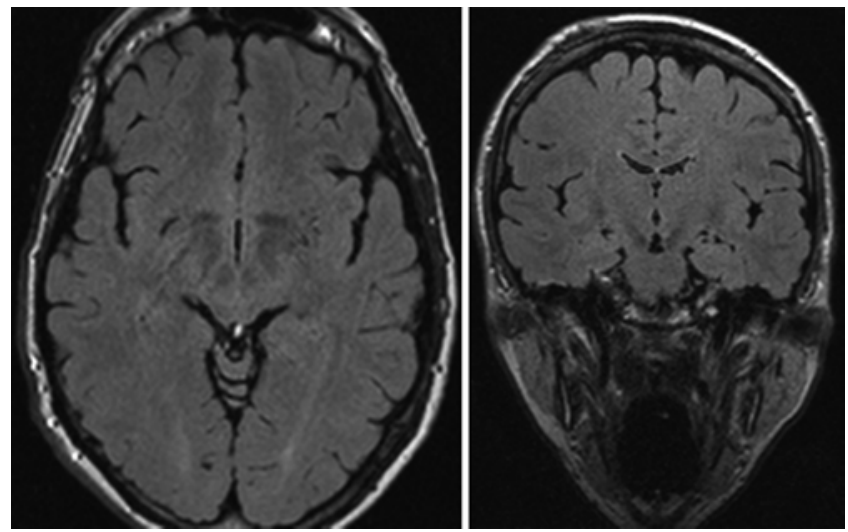

FIG. 3. Axial (left) and coronal (right) FLAIR images at $1.5 \mathrm{~T}$ through the center of the STN.

were more clearly discernable on T2 FSE, suggesting a combination of both T2 FSE and STIR would be able to delineate all the borders of the STN..$^{24}$ Unfortunately, evidence suggests that conspicuity of the STN in patients with Parkinson's disease may decrease with age using the STIR technique. ${ }^{41}$ Given that these patients are often elderly, this sequence may not provide sufficient contrast to the STN for direct targeting.

Newer sequences such as FGATIR have shown promise because of excellent CNR at the level of the STN. ${ }^{53}$ In comparison with T1 and T2W FLAIR sequences, FGATIR demonstrated 3 times the CNR when comparing the STN to the SN. Qualitative analysis also showed that all STN borders, except for the border with the posterior limb of the internal capsule, were better visualized with FGATIR. Given that this pilot study was performed on only 3 patients, larger patient cohorts and comparison with newer imaging techniques will be needed to verify the role of FGATIR in clinical practice. Nonetheless, this technique represents a promising development in the use of IR imaging for visualizing the STN in the future.

\section{Susceptibility-Based Sequences}

All susceptibility sequences are based on SWI and use a $3 \mathrm{D}$ velocity-compensated gradient echo (GRE) sequence to visualize deep brain structures. ${ }^{20}$ This method exploits the magnetic susceptibility differences between tissues and has been of particular interest in imaging the STN because of a demonstrated increase in iron content of the STN as patients age and in the setting of neurodegenerative diseases. ${ }^{8,14}$ This inherent paramagnetic nature of the STN means that good contrast can be achieved between the STN and its surrounding structures.

Using postprocessing techniques, a single SWI acquisition can provide 3 sets of images. ${ }^{29,57}$ These include a T2 image, providing magnitude details $(\mathrm{T} 2 * \mathrm{WI})$; a susceptibility-weighted phase image, providing phase information; and a combined image (SWI), which has traditionally been referred to as a venogram given its excellent depiction of vessels that contain deoxyhemoglobin. ${ }^{57}$ Each of these sequences has been studied individually for identification of the STN. 
T2-Weighted Magnitude Imaging. Iron-containing tissue, such as the STN in elderly patients with neurodegenerative disease, appears darker on $\mathrm{T} 2 * \mathrm{WI}$ when compared with T2WI. This is because T2*WI incorporates traditional $\mathrm{T} 2$ relaxation with magnetic field inhomogeneity caused by the tissue iron deposits. Tissue ferritin and hemosiderin causes rapid proton dephasing and transverse magnetization degradation. The STN thus appears relatively darker. It is seen in GRE image sequences as the transverse relaxation caused by field inhomogeneity is nullified in FSE sequences. ${ }^{10}$

The clinically relevant $\mathrm{T} 2 *$ sequences are mostly spoiled GRE sequences, including fast low-angle shot (FLASH) and postexcitation refocused GRE. ${ }^{10}$ Of these sequences, T2* FLASH has been the most studied as it allows images to be obtained rapidly and with greater SNR. ${ }^{16}$ T2* FLASH has been compared directly to various T1 and T2 sequences and to SWI at both $3 \mathrm{~T}$ and 7 $\mathrm{T}^{22,23}$ The ability to delineate the STN, in particular, from the ZI and the SN was assessed. At both $3 \mathrm{~T}$ and $7 \mathrm{~T}$, coronal T2* FLASH sequences provided the best visualization of the STN. Interpretation of these results by DBS surgeons remains difficult, however, given these studies were performed in healthy volunteers, without a head frame, and without testing for geometrical accuracy.

Susceptibility-Weighted Phase Imaging. Susceptibility-weighted phase images have been shown to have excellent ability to delineate the STN from the SN and the ZI. ${ }^{57}$ Phase images are not dependent on $\mathrm{T} 1$ and $\mathrm{T} 2$ relaxation parameters and are less affected by field inhomogeneity secondary to high field strength, which is its main advantages over T2*WI. ${ }^{49}$

Qualitative analysis of STN visibility using SWI, SWPI, and T2* showed that SWPI had almost double the visibility compared with SWI and 3.5 times the visibility compared with T2*WI. ${ }^{57}$ Comparison of T2, T2*, SWI, SWPI, and QSM showed phase images were second only to QSM for visualization of the STN, with good interrater reliability and a median 5 times increase in CNR compared with T2WI. ${ }^{29}$

Susceptibility-Weighted Imaging. Susceptibility-weighted images (Fig. 4) are a combination of phase and magnitude images which produces an enhanced-contrast magnitude image that is particularly sensitive to hemorrhage, calcium, iron storage, and slow venous blood containing deoxyhemoglobin. ${ }^{17}$ This sequence has been shown to have a wide variety of clinical applications including the identification of cerebral arteriovenous malformations, tumors, venous disease, trauma, and functional brain imaging. ${ }^{20}$

SWI has repeatedly been demonstrated to be better than T2WI for visualization of the STN, with a statistically significant increase in CNR even at low field strength. ${ }^{29,36}$ The other advantage with SWI in DBS applications is that it enhances visualization of deep cerebral veins and transparenchymal vessels to assist in preoperative planning of DBS lead trajectory.7,40

SWI provides good CNR at the level of the STN, but evidence suggests it does not perform as well as phase and T2* images, particularly at higher field strength. Direct
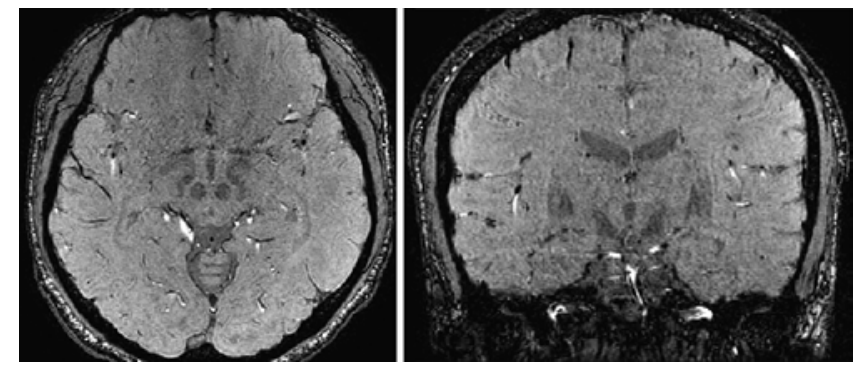

FIG. 4. Axial (left) and coronal (right) susceptibility-weighted MR images through the center of the STN. Courtesy of Dr. Tian Liu, MedImageMetric LLC, New York, New York, and Dr. Yi Wang, Biomedical Engineering and Radiology, Cornell University, New York, New York.

comparison of SWI with T2* FLASH images at $3 \mathrm{~T}$ and 7 T showed T2* FLASH 2D provided superior contrast for the STN. ${ }^{22,23}$ Phase images have also been shown to provide better delineation of the STN, with visualization scores of 3.47 and 2.03 out of 5 for phase images and SWI, respectively. ${ }^{57}$ Quantitative analysis demonstrates a higher CNR for phase imaging in comparison with SWI when viewing the STN and SN. ${ }^{29}$

Disadvantages of Susceptibility-Based Imaging Techniques. There are disadvantages with susceptibility-based imaging techniques, including signal loss, distortion, and local field inhomogeneity, particularly at high field strength. ${ }^{1,17,57}$ This can often blur the edges of the STN and cause it to appear larger.

Assuming iron in the STN is uniformly distributed, the susceptibility signal of GRE imaging would not accurately represent the STN's borders. This is because the magnetic field produced by the MRI scanner (Bo) induces local magnetic fields in paramagnetic tissue (Fig. 5). These local magnetic fields extend beyond the borders of the STN and can cause protons in surrounding tissue to relax faster, leading to decreased signal in these areas. This means that in susceptibility imaging the STN may appear to originate from surrounding non-STN tissue. This is known as a nonlocal susceptibility effect. It is further complicated by the fact that iron is not uniformly distributed in the STN, and thus certain borders may be more exaggerated than others. GRE imaging, in general, is predisposed to nonlocal susceptibility effects, also known as blooming artifacts, which represent a significant drawback of all susceptibility-based imaging.

Occurring at the surface, blooming artifacts appear to arise perpendicular to the magnetic field. ${ }^{27}$ Given the STN lies obliquely in 3 planes and is a small structure, these blooming artifacts need quantification and correction prior to accurate direct targeting of the STN. Image reconstruction methods such as QSM represent a way to address this problem.

Quantitative Susceptibility Mapping. QSM (Fig. 6) is a post-image processing technique that can be used on any GRE phase image. ${ }^{27}$ The goals of QSM are to reduce orientation dependency of the target brain tissue, thus reducing blooming artifacts, and to provide a more direct measurement of intrinsic tissue magnetic properties and iron content, which allows for better delineation of 


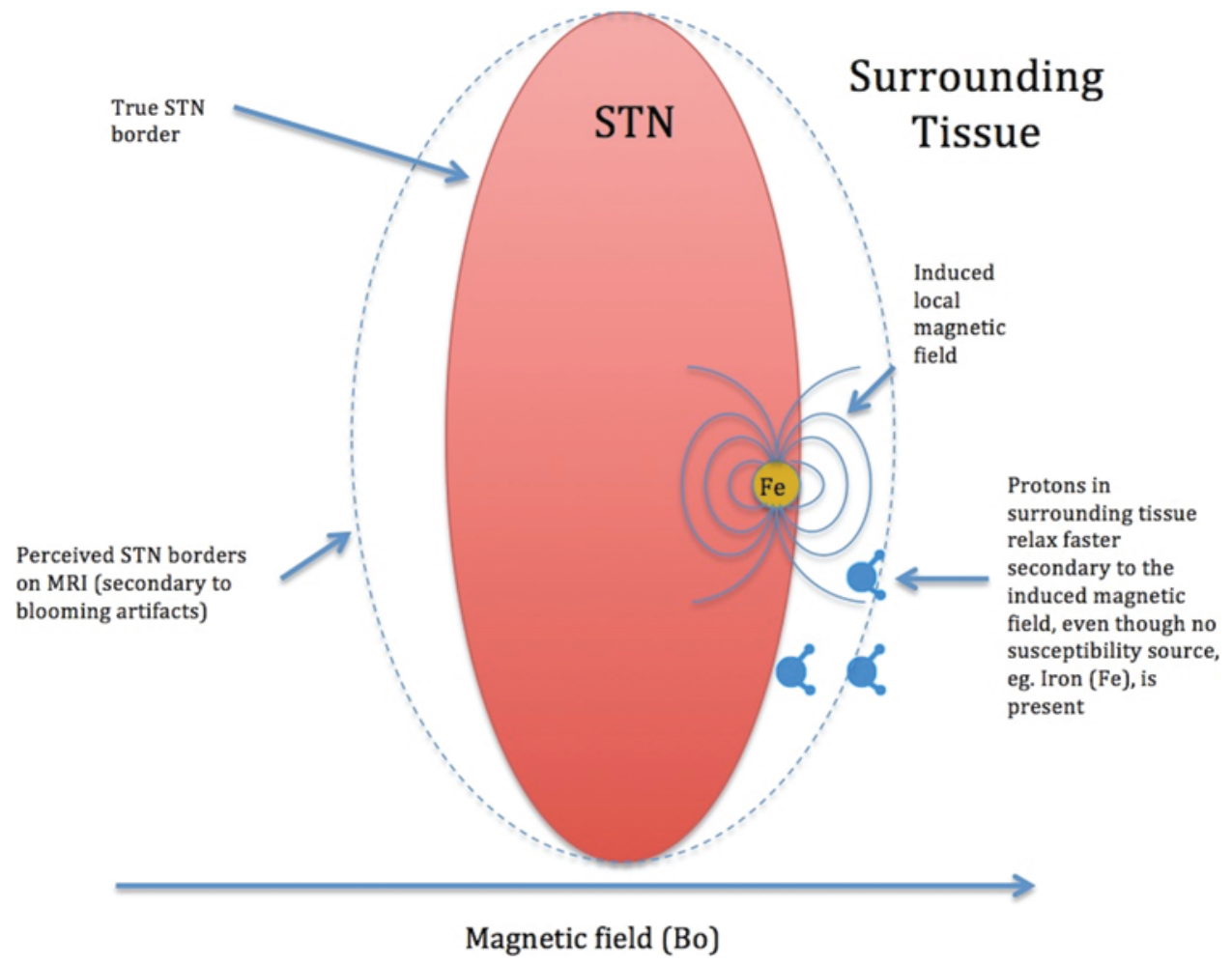

FIG. 5. Diagrammatic representation of nonlocal susceptibility effects (blooming artifacts) and the STN. Iron (Fe) within the STN produces a local magnetic field when under the influence of the main magnetic field (Bo). This local magnetic field induces relaxation of protons in surrounding tissues, producing a susceptibility effect even though no susceptibility source is present. This blurs the borders of the STN and can cause them to appear larger than they really are. Copyright Arjun Chandran. Used with permission.

the STN from the SN and other proximal iron-rich structures. ${ }^{13,27,42,49}$

Reduced Object Orientation Dependency. The susceptibility effect is nonlocal in GRE imaging. This is because the unusual shape of the dipole kernel makes the field map inhomogeneous, even when the underlying tissue is homogenous: a phenomenon predicted by Maxwell's equations for static magnetism. ${ }^{27}$ These nonlocal effects produce characteristic blooming artifacts.

Dipole deconvolution is a method used in QSM and directly reduces blooming artifacts, providing a clearer
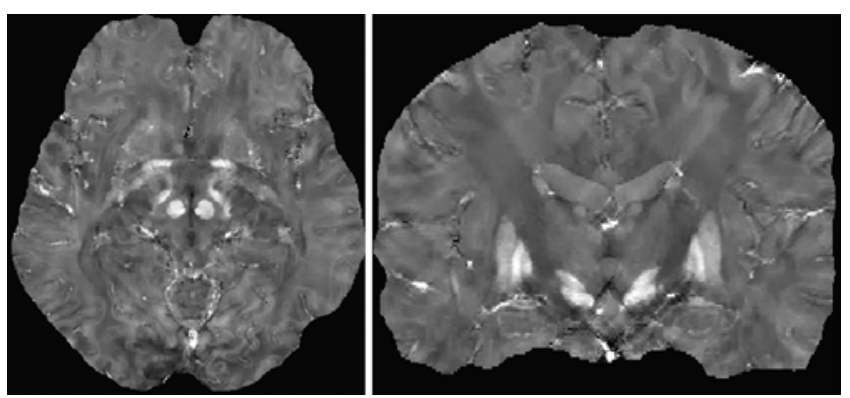

FIG. 6. Axial (left) and coronal (right) QSM images through the center of the STN. Courtesy of Dr. Tian Liu, MedlmageMetric LLC, New York, New York, and Dr. Yi Wang, Biomedical Engineering and Radiology, Cornell University, New York, New York. picture of tissue susceptibility and magnetic properties. This has been directly measured through experimentation with copper sulfate phantoms, where it was demonstrated that blooming artifacts are most notable at the surfaces of an object and perpendicular to the magnetic field. ${ }^{27}$ Using QSM reduced blooming artifacts and allows for clearer definition of the phantom borders regardless of orientation. This is of particular importance in considering imaging of the STN given its oblique orientation. ${ }^{3}$ It may also mean accurate depiction of the STN would be otherwise highly dependent on something as simple as head orientation within the scanner. ${ }^{42}$

More Accurate Measurement of Brain Iron Concentration. The presence of brain iron has been shown to be the predominant factor in magnetic susceptibility in gray matter. ${ }^{26}$ However, iron concentration within the STN is not homogenously distributed, and studies have suggested increased concentration inferiorly. ${ }^{14}$ This combined with nonlocal phase changes present in susceptibility-based imaging means that $\mathrm{T} 2 *$ hypointensity and phase contrast are not directly reflective of local tissue iron concentration. ${ }^{27}$

QSM produces a more linear correlation of tissue iron concentration. ${ }^{27}$ This has been corroborated by postmortem evaluation of deep gray matter structures. ${ }^{26}$ More accurate estimation of gray matter iron concentrations allows for better discrimination of surrounding iron-rich gray matter structures, including the $\mathrm{SN} .{ }^{42}$ 
There is only one study directly comparing QSM imaging with other techniques for viewing the STN (T2, T2*WI, R2*, SWPI, and SWI) in both healthy volunteers and neurological patients undergoing DBS. ${ }^{29}$ The study demonstrated that the STN was most clearly delineated from the ZI superiorly and the SN inferiorly using QSM, and that QSM directly reduced blooming artifacts produced by GRE sequences. The CNR was 6.4 times higher on QSM than on conventional T2WI for differentiating the STN from surrounding structures.

QSM has not been verified for preoperative planning in DBS to date. In particular, the geometrical accuracy of this technique needs further quantification. Postprocessing is technically demanding and may require a significant amount of time to calculate the images. ${ }^{44,58}$ An online reconstruction technique has shown promise in addressing this practical limitation, reducing the image construction time to less than 30 seconds on standard computers. ${ }^{45}$

\section{Discussion}

The placement of DBS leads was originally performed using ventriculographic guidance, which provided distortion-free demonstration of the anterior and posterior commissure. This technique is, however, invasive, may increase the length of hospitalization, and is necessarily indirect as structures cannot not be visualized. ${ }^{34}$

The advent of CT improved the safety and convenience of stereotactic imaging but still required an indirect approach. This ongoing reliance on atlases may have been a source of variability in clinical results. For example, the Schaltenbrand and Wahren atlases ${ }^{43}$ represent a very small sample of the normal adult population, and moreover did not use individuals with Parkinson's disease. ${ }^{28}$

MRI allows direct visualization of the STN; however, direct targeting has only been made possible recently, with technological advances in hardware, increased field strength, and new sequences. Even as recently as 15 years ago stereotactic targeting was necessarily indirect as MRI technology and subsequent image quality had not advanced sufficiently for neurosurgeons to feel comfortable directly targeting the STN. As technology advances and images improve, direct targeting is becoming more feasible and readily acceptable.

Over time, pulse sequences have advanced from basic SE sequences such as T1 and T2 to newer SE and susceptibility-based sequences that provide signal contrast based on differences in brain tissue composition. Despite the availability of these newer sequences, their use in stereotactic surgery is currently limited.

T2WI and IR imaging remain the predominant sequences used and have been for many years. Short scanning times, a perceived lack of evidence-based alternatives, and neurosurgeons' familiarity with interpreting these images may explain their ongoing popularity.

High-quality, distortion-free images are paramount for neurosurgeons. There are 2 primary concerns with currently used sequences. The first is geometrical distortion secondary to both the presence of a paramagnetic head frame and the nature of the sequence itself. Distortion has led some groups to coregister MRI to CT in the hope of reducing geometrical inaccuracy inherent in MRI techniques. There is no strong evidence to support this, with some studies demonstrating the CT/MRI fusion process may, in fact, introduce geometrical error. ${ }^{55}$ Distortion correction algorithms have also been used; however, the techniques are imperfect and less ideal than producing direct distortion-free imaging sequences.

The second concern for neurosurgeons using direct targeting techniques is poor image quality. Attempts to improve image quality with currently used sequences have led some groups to image while the patient is immobilized using general anesthesia for long acquisition times. This, for example, allows for an increase in the number of signal averages (NSA), which may improve the CNR. The major limitation in improving image quality remains the requirement to use a transmit-receive head coil with the paramagnetic head frame.

The authors currently use stereotactic head frame T2WI at $3 \mathrm{~T}$ with a high NSA under general anesthesia in an attempt to improve STN visualization (Table 1). This

TABLE 1. Literature-based summary of imaging sequence parameters commonly used for STN assessment*

\begin{tabular}{lccccc}
\hline \multicolumn{1}{c}{ Parameter } & T2 FSE & PSIR & FGATIR & Coronal T2* FLASH 2D & SWI \\
\hline Field strength & $3 \mathrm{~T}$ & $1.5 \mathrm{~T}$ & $3 \mathrm{~T}$ & $3 \mathrm{~T}$ & $1.5 \mathrm{~T}$ \\
\hline Matrix & $384 \times 384$ & $230 \times 230$ & $320 \times 256$ & $384 \times 384$ & $256 \times 256$ \\
\hline FOV, mm & $260 \times 260$ & $230 \times 230$ & $256 \times 192$ & $192 \times 192$ & $250 \times 250$ \\
\hline Slice thickness, $\mathrm{mm}$ & 1.0 & 2.0 & 1.0 & 2.5 & 2.0 \\
\hline TE, msec & 100 & 10 & 4.39 & 30 & 40 \\
\hline TR, msec & 10,672 & 4000 & 3000 & 625 & - \\
\hline TI msec & - & 200 & 409 & 30 & 90 \\
\hline Flip angle, degrees & $90 / 130$ & - & - & $40 \mathrm{~Hz}$ & $21 \mathrm{kHz}$ \\
\hline Bandwidth & $250 \mathrm{~Hz}$ & - & $130 \mathrm{~Hz}$ & 1 & - \\
\hline Mean no. of signals & 8 & - & - & $32 \mathrm{channel}$ & Quadrature \\
\hline Head coil & Transmit-receive & Quadrature & Quadrature & & - \\
\hline
\end{tabular}

FOV = field of view; $T E=$ echo time.

* Data for the different sequences were derived from the following references: Thani et al. ${ }^{55}$ (T2 FSE), Ishimori et al. ${ }^{21}$ (PSIR), Sudhyadhom et al. ${ }^{53}$ (FGATIR), Kerl et al. ${ }^{22}$ (Coronal T2* FLASH 2D), O'Gorman et al. ${ }^{36}$ (SWI). 
is after many years of experimenting with both T2 and IR sequences and image parameters. Despite our best efforts at improving image quality with commonly used sequences, the STN remains difficult to visualize, especially in relation to the SN. Our experience suggests that despite gradual improvements in MRI technology there is still a need for better imaging.

Improvements in imaging can be achieved using a variety of methods. The first is to improve the field strength. This allows a greater CNR and SNR. Increased distortion at higher field strength remains a concern, however. The second method is to improve the head coil used. Unfortunately the use of a head frame limits head coil design to transmit-receive. The use of frameless or robotic techniques to bypass this limitation may become increasingly important in the future. ${ }^{25}$ The third method is to alter the pulse sequence used, but this requires neurosurgeons to spend time optimizing the new sequences and gaining confidence with its use.

Broadly speaking, all imaging sequences discussed fall into 2 categories. The first are SE techniques based on the precession of water molecules, which include the sequences commonly used worldwide such as T2WI, IR imaging, and DTI. Tissue contrast in these sequences is based on differences in decaying rates, which is often small as water is relatively uniformly distributed throughout the tissue. These sequences have been extensively investigated for imaging the STN; however, given the lack of specificity for the target tissue being imaged, they are often deficient in the level of contrast needed to accurately target it.

The second group of sequences are the newer susceptibility-based techniques including SWI, SWPI, and T2*WI, which take into account the underlying composition of the brain tissue, which, most importantly in the case of the STN, includes iron deposits. Only certain metallic elements of biomolecules, such as highly paramagnetic iron, have unpaired electrons that are very selectively deposited in cerebral structures, ${ }^{26}$ which provide a significant advantage as pulse sequences that are able to take this into account provide excellent contrast. The disadvantages of these sequences are the presence of orientation dependency and nonlocal effects in GRE imaging. This produces blooming artifacts that blur the borders of the STN. For neurosurgeons this means excellent quality images with an improved ability to delineate all STN borders but a strong potential for lead placement distant to the desired target.

Accurate placement of DBS leads requires both good contrast at the level of the STN to plan for targeting, and accurate boundaries to maximize therapeutic benefit and minimize stimulation side effects. All of the above sequences thus require a trade-off between contrast and accurate demarcation of the STN's boundaries. Although very good images of the STN have been generated with susceptibilitybased techniques, there are no reliable published data to suggest that they can be accurately used stereotactically. Blooming artifacts may produce such significant distortion that the accuracy of electrode placement is affected.

QSM represents the most exciting advance in imaging of the STN. Comparison studies show it provides better CNR than susceptibility-based imaging, and early phantom studies suggest a reduction in blooming artifacts, giv- ing more accurate boundaries of the STN. The difficulty in this technique lies in the ability for small centers to use it given the high computational load required to postprocess the images. Faster image reconstruction ${ }^{45}$ may soon become available so that research groups can begin to assess its suitability for STN stereotaxis.

\section{Conclusions}

DBS of the STN is difficult both because of geometrical distortion and poor resolution on currently available MRI sequences used in direct targeting. New MRI sequences have shown promise in improving STN contrast and better defining STN borders from the SN and ZI. Susceptibilitybased imaging techniques and image reconstruction methods may represent the way forward to producing highquality, distortion-free images with excellent STN edge detection that neurosurgeons can use to accurately and reliably target their electrodes.

\section{Acknowledgments}

We would like to thank Drs. Tian Liu and Yi Wang for providing Figs. 4 and 6.

\section{References}

1. Abosch A, Yacoub E, Ugurbil K, Harel N: An assessment of current brain targets for deep brain stimulation surgery with susceptibility-weighted imaging at 7 tesla. Neurosurgery 67:1745-1756, 2010

2. Andrade-Souza YM, Schwalb JM, Hamani C, Eltahawy H, Hoque T, Saint-Cyr J, et al: Comparison of three methods of targeting the subthalamic nucleus for chronic stimulation in Parkinson's disease. Neurosurgery 56 (2 Suppl):360-368, 2005

3. Ashkan K, Blomstedt P, Zrinzo L, Tisch S, Yousry T, Limousin-Dowsey $\mathrm{P}$, et al: Variability of the subthalamic nucleus: the case for direct MRI guided targeting. Br J Neurosurg 21:197-200, 2007

4. Barkhoudarian G, Klochkov T, Sedrak M, Frew A, Gorgulho A, Behnke E, et al: A role of diffusion tensor imaging in movement disorder surgery. Acta Neurochir (Wien) 152:2089-2095, 2010

5. Basser PJ, Pajevic S, Pierpaoli C, Duda J, Aldroubi A: In vivo fiber tractography using DT-MRI data. Magn Reson Med 44:625-632, 2000

6. Bejjani BP, Dormont D, Pidoux B, Yelnik J, Damier P, Arnulf I, et al: Bilateral subthalamic stimulation for Parkinson's disease by using three-dimensional stereotactic magnetic resonance imaging and electrophysiological guidance. J Neurosurg 92:615-625, 2000

7. Ben-Haim S, Asaad WF, Gale JT, Eskandar EN: Risk factors for hemorrhage during microelectrode-guided deep brain stimulation and the introduction of an improved microelectrode design. Neurosurgery 64:754-763, 2009

8. Brass SD, Chen NK, Mulkern RV, Bakshi R: Magnetic resonance imaging of iron deposition in neurological disorders. Top Magn Reson Imaging 17:31-40, 2006

9. Brunenberg EJ, Platel B, Hofman PA, Ter Haar Romeny BM, Visser-Vandewalle V: Magnetic resonance imaging techniques for visualization of the subthalamic nucleus. J Neurosurg 115:971-984, 2011

10. Chavhan GB, Babyn PS, Thomas B, Shroff MM, Haacke EM: Principles, techniques, and applications of T2*-based MR imaging and its special applications. Radiographics 29:1433-1449, 2009 
11. Cheng $\mathrm{CH}$, Huang HM, Lin HL, Chiou SM: $1.5 \mathrm{~T}$ versus $3 \mathrm{~T}$ MRI for targeting subthalamic nucleus for deep brain stimulation. Br J Neurosurg 28:467-470, 2014

12. Danish SF, Jaggi JL, Moyer JT, Finkel L, Baltuch GH: Conventional MRI is inadequate to delineate the relationship between the red nucleus and subthalamic nucleus in Parkinson's disease. Stereotact Funct Neurosurg 84:12-18, 2006

13. de Rochefort L, Liu T, Kressler B, Liu J, Spincemaille P, Lebon V, et al: Quantitative susceptibility map reconstruction from MR phase data using bayesian regularization: validation and application to brain imaging. Magn Reson Med 63:194-206, 2010

14. Dormont D, Ricciardi KG, Tandé D, Parain K, Menuel C, Galanaud D, et al: Is the subthalamic nucleus hypointense on T2-weighted images? A correlation study using MR imaging and stereotactic atlas data. AJNR Am J Neuroradiol 25:1516-1523, 2004

15. Elmaoğlu M, Çelik A: MRI Handbook: MR Physics, Patient Positioning, and Protocols. New York: Springer, 2012

16. Frahm J, Haase A, Matthaei D: Rapid NMR imaging of dynamic processes using the FLASH technique. Magn Reson Med 3:321-327, 1986

17. Gasparotti R, Pinelli L, Liserre R: New MR sequences in daily practice: susceptibility weighted imaging. A pictorial essay. Insights Imaging 2:335-347, 2011

18. Guehl D, Edwards R, Cuny E, Burbaud P, Rougier A, Modolo J, et al: Statistical determination of the optimal subthalamic nucleus stimulation site in patients with Parkinson disease. J Neurosurg 106:101-110, 2007

19. Gutman DA, Holtzheimer PE, Behrens TE, Johansen-Berg H, Mayberg HS: A tractography analysis of two deep brain stimulation white matter targets for depression. Biol Psychiatry 65:276-282, 2009

20. Haacke EM, Xu Y, Cheng YC, Reichenbach JR: Susceptibility weighted imaging (SWI). Magn Reson Med 52:612-618, 2004

21. Ishimori T, Nakano S, Mori Y, Seo R, Togami T, Masada T, et al: Preoperative identification of subthalamic nucleus for deep brain stimulation using three-dimensional phase sensitive inversion recovery technique. Magn Reson Med Sci 6:225-229, 2007

22. Kerl HU, Gerigk L, Pechlivanis I, Al-Zghloul M, Groden C, Nölte I: The subthalamic nucleus at 3.0 Tesla: choice of optimal sequence and orientation for deep brain stimulation using a standard installation protocol: clinical article. J Neurosurg 117:1155-1165, 2012

23. Kerl HU, Gerigk L, Pechlivanis I, Al-Zghloul M, Groden C, Nölte IS: The subthalamic nucleus at 7.0 Tesla: evaluation of sequence and orientation for deep-brain stimulation. Acta Neurochir (Wien) 154:2051-2062, 2012

24. Kitajima M, Korogi Y, Kakeda S, Moriya J, Ohnari N, Sato T, et al: Human subthalamic nucleus: evaluation with high-resolution MR imaging at 3.0 T. Neuroradiology 50:675-681, 2008

25. Konrad PE, Neimat JS, Yu H, Kao CC, Remple MS, D'Haese $\mathrm{PF}$, et al: Customized, miniature rapid-prototype stereotactic frames for use in deep brain stimulator surgery: initial clinical methodology and experience from 263 patients from 2002 to 2008. Stereotact Funct Neurosurg 89:34-41, 2011

26. Langkammer C, Schweser F, Krebs N, Deistung A, Goessler W, Scheurer E, et al: Quantitative susceptibility mapping (QSM) as a means to measure brain iron? A post mortem validation study. Neuroimage 62:1593-1599, 2012

27. Li J, Chang S, Liu T, Wang Q, Cui D, Chen X, et al: Reducing the object orientation dependence of susceptibility effects in gradient echo MRI through quantitative susceptibility mapping. Magn Reson Med 68:1563-1569, 2012

28. Littlechild P, Varma TR, Eldridge PR, Fox S, Forster A, Fletcher N, et al: Variability in position of the subthalamic nucleus targeted by magnetic resonance imaging and microelectrode recordings as compared to atlas co-ordinates. Stereotact Funct Neurosurg 80:82-87, 2003

29. Liu T, Eskreis-Winkler S, Schweitzer AD, Chen W, Kaplitt MG, Tsiouris AJ, et al: Improved subthalamic nucleus depiction with quantitative susceptibility mapping. Radiology 269:216-223, 2013

30. Lu H, Nagae-Poetscher LM, Golay X, Lin D, Pomper M, van Zijl PC: Routine clinical brain MRI sequences for use at 3.0 Tesla. J Magn Reson Imaging 22:13-22, 2005

31. Mallet L, Schüpbach M, N'Diaye K, Remy P, Bardinet E, Czernecki V, et al: Stimulation of subterritories of the subthalamic nucleus reveals its role in the integration of the emotional and motor aspects of behavior. Proc Natl Acad Sci U S A 104:10661-10666, 2007

32. Manova ES, Habib CA, Boikov AS, Ayaz M, Khan A, Kirsch WM, et al: Characterizing the mesencephalon using susceptibility-weighted imaging. AJNR Am J Neuroradiol 30:569-574, 2009

33. Massey LA, Miranda MA, Zrinzo L, Al-Helli O, Parkes HG, Thornton JS, et al: High resolution MR anatomy of the subthalamic nucleus: imaging at $9.4 \mathrm{~T}$ with histological validation. Neuroimage 59:2035-2044, 2012

34. Menuel C, Garnero L, Bardinet E, Poupon F, Phalippou D, Dormont D: Characterization and correction of distortions in stereotactic magnetic resonance imaging for bilateral subthalamic stimulation in Parkinson disease. J Neurosurg 103:256-266, 2005

35. Mori S, Crain BJ, Chacko VP, van Zijl PC: Three-dimensional tracking of axonal projections in the brain by magnetic resonance imaging. Ann Neurol 45:265-269, 1999

36. O'Gorman RL, Shmueli K, Ashkan K, Samuel M, Lythgoe DJ, Shahidiani A, et al: Optimal MRI methods for direct stereotactic targeting of the subthalamic nucleus and globus pallidus. Eur Radiol 21:130-136, 2011

37. Patil PG, Conrad EC, Aldridge JW, Chenevert TL, Chou KL: The anatomical and electrophysiological subthalamic nucleus visualized by 3-T magnetic resonance imaging. Neurosurgery 71:1089-1095, 2012

38. Plaha P, Ben-Shlomo Y, Patel NK, Gill SS: Stimulation of the caudal zona incerta is superior to stimulation of the subthalamic nucleus in improving contralateral parkinsonism. Brain 129:1732-1747, 2006

39. Poupon C, Clark CA, Frouin V, Régis J, Bloch I, Le Bihan $\mathrm{D}$, et al: Regularization of diffusion-based direction maps for the tracking of brain white matter fascicles. Neuroimage 12:184-195, 2000

40. Rauscher A, Sedlacik J, Barth M, Haacke EM, Reichenbach JR: Noninvasive assessment of vascular architecture and function during modulated blood oxygenation using susceptibility weighted magnetic resonance imaging. Magn Reson Med 54:87-95, 2005

41. Sarkar SN, Sarkar PR, Papavassiliou E: Subthalamic nuclear tissue contrast in inversion recovery MRI decreases with age in medically refractory Parkinson's disease. J Neuroimaging 25:303-306, 2015

42. Schäfer A, Forstmann BU, Neumann J, Wharton S, Mietke A, Bowtell R, et al: Direct visualization of the subthalamic nucleus and its iron distribution using high-resolution susceptibility mapping. Hum Brain Mapp 33:2831-2842, 2012

43. Schaltenbrand G, Wahren W, Hassler R: Atlas for Stereotaxy of the Human Brain, ed 2. Stuttgart: Thieme, 1977

44. Schweser F, Deistung A, Lehr BW, Reichenbach JR: Differentiation between diamagnetic and paramagnetic cerebral lesions based on magnetic susceptibility mapping. Med Phys 37:5165-5178, 2010

45. Schweser F, Deistung A, Sommer K, Reichenbach JR: Toward online reconstruction of quantitative susceptibil- 
ity maps: superfast dipole inversion. Magn Reson Med 69:1582-1594, 2013

46. Sedrak M, Gorgulho A, Bari A, Behnke E, Frew A, Gevorkyan I, et al: Diffusion tensor imaging (DTI) and colored fractional anisotropy (FA) mapping of the subthalamic nucleus (STN) and the globus pallidus interna (GPi). Acta Neurochir (Wien) 152:2079-2084, 2010

47. Sedrak M, Gorgulho A, De Salles AF, Frew A, Behnke E, Ishida $\mathrm{W}$, et al: The role of modern imaging modalities on deep brain stimulation targeting for mental illness. Acta Neurochir Suppl 101:3-7, 2008

48. Shields DC, Gorgulho A, Behnke E, Malkasian D, DeSalles AA: Contralateral conjugate eye deviation during deep brain stimulation of the subthalamic nucleus. J Neurosurg 107:37-42, 2007

49. Shmueli K, de Zwart JA, van Gelderen P, Li TQ, Dodd SJ, Duyn JH: Magnetic susceptibility mapping of brain tissue in vivo using MRI phase data. Magn Reson Med 62:1510 1522,2009

50. Simon SL, Douglas P, Baltuch GH, Jaggi JL: Error analysis of MRI and Leksell stereotactic frame target localization in deep brain stimulation surgery. Stereotact Funct Neurosurg 83:1-5, 2005

51. Starr PA, Christine CW, Theodosopoulos PV, Lindsey N, Byrd D, Mosley A, et al: Implantation of deep brain stimulators into the subthalamic nucleus: technical approach and magnetic resonance imaging-verified lead locations. J Neurosurg 97:370-387, 2002

52. Starr PA, Vitek JL, DeLong M, Bakay RA: Magnetic resonance imaging-based stereotactic localization of the globus pallidus and subthalamic nucleus. Neurosurgery 44:303314, 1999

53. Sudhyadhom A, Haq IU, Foote KD, Okun MS, Bova FJ: A high resolution and high contrast MRI for differentiation of subcortical structures for DBS targeting: the Fast Gray Matter Acquisition T1 Inversion Recovery (FGATIR). Neuroimage 47 (Suppl 2):T44-T52, 2009

54. Thani NB, Bala A, Lind CR: Accuracy of magnetic resonance imaging-directed frame-based stereotaxis. Neurosurgery 70 (1 Suppl Operative):114-124, 2012
55. Thani NB, Bala A, Swann GB, Lind CR: Accuracy of postoperative computed tomography and magnetic resonance image fusion for assessing deep brain stimulation electrodes. Neurosurgery 69:207-214, 2011

56. Tsai ST, Lin SH, Lin SZ, Chen JY, Lee CW, Chen SY: Neuropsychological effects after chronic subthalamic stimulation and the topography of the nucleus in Parkinson's disease. Neurosurgery 61:E1024-1030, 2007

57. Vertinsky AT, Coenen VA, Lang DJ, Kolind S, Honey CR, Li $\mathrm{D}$, et al: Localization of the subthalamic nucleus: optimization with susceptibility-weighted phase MR imaging. AJNR Am J Neuroradiol 30:1717-1724, 2009

58. Wu B, Li W, Guidon A, Liu C: Whole brain susceptibility mapping using compressed sensing. Magn Reson Med 67:137-147, 2012

59. Zonenshayn M, Rezai AR, Mogilner AY, Beric A, Sterio D, Kelly PJ: Comparison of anatomic and neurophysiological methods for subthalamic nucleus targeting. Neurosurgery 47:282-294, 2000

\section{Disclosure}

The authors report no conflict of interest concerning the materials or methods used in this study or the findings specified in this paper.

\section{Author Contributions}

Conception and design: Lind, Chandran. Acquisition of data: Chandran. Analysis and interpretation of data: Lind, Chandran. Drafting the article: Chandran. Critically revising the article: Lind, Bynevelt. Reviewed submitted version of manuscript: all authors. Approved the final version of the manuscript on behalf of all authors: Lind. Study supervision: Lind.

\section{Correspondence}

Christopher Lind, Neurosurgical Service of Western Australia, 1st Fl., G Block, Sir Charles Gairdner Hospital, Nedlands, Perth, WA 6009, Australia. email: christopher.lind@health.gov.wa.au. 